\title{
Maintien d'un gène létal dans une population: le cas des chats sans queue de l'ile de Man
}

\author{
Jean GÉNERMONT \\ Laboratoire de Zoologie 2, Université de Paris-Sud \\ F-91405 Orsay Cedex
}

\begin{abstract}
Résumé
Un modèle déterministe fondé sur l'existence de distorsions des ségrégations mendéliennes et de différences de fécondité entre diverses catégories de couples permet d'expliquer, dans certaines conditions, le maintien d'un allèle létal dans une population. Les prédictions en sont discutées dans la perspective d'une application à la population de chats de l'Ile de Man : le maintien de l'allèle $M$ (qui, à l'état hétérozygote, détermine l'absence de queue) peut être compris sans faire appel à une sélection zygotique post-natale.
\end{abstract}

Le phénotype «sans queue » est exceptionnel chez le chat domestique. On le connaît néanmoins depuis plus d'un siècle, à fréquence non négligeable, dans la population de l'Ile de Man. On admet qu'il se manifeste chez des animaux hétérozygotes pour un couple d'allèles autosomal, désigné par $M-m, m$ étant l'allèle sauvage, le génotype homozygote $M M$ étant létal. Si cette interprétation est correcte, elle pose le problème du maintien d'un gène létal dans une population.

Le mécanisme le plus simple qui puisse être envisagé est celui d'un avantage sélectif de l'hétérozygote dû à une intervention humaine : les habitants de l'Ile de Man auraient une préférence pour les chats sans queue (TodD, GarRad \& Blumenberg, 1979). Cependant, dans un récent travail, AdALsteinsson (1980) suggère une autre hypothèse, fondée sur l'analyse des ségrégations observées parmi les progénitures des trois catégories de couples $M / m \times M / m$, \& $M / m \times$ o $m / m$ et $q \mathrm{~m} / \mathrm{m} \times \hat{\alpha} \mathrm{M} / \mathrm{m}$ : ces ségrégations sont en effet significativement différentes des ségrégations attendues, $2 / 3-1 / 3$ dans le premier cas, $1 / 2-1 / 2$ dans les deux autres. Pour expliquer ces déviations, deux mécanismes sont proposés : une sélection gamétique agissant sur les spermatozoïdes et une dérive méiotique lors de la gamétogenèse femelle (pour un ovule formé par une femelle $M / m$, la probabilité de recevoir l'allèle $M$ ne serait pas obligatoirement $1 / 2$, mais dépendrait du génotype du spermatozoïde fécondant, dont la pénétration a lieu alors que la méiose n'est pas encore terminée).

Cette hypothèse n'est évidemment pas la seule qui puisse rendre compte d'une distorsion des ségrégations mendéliennes. On peut penser par exemple à une sélection 
zygotique intra-utérine. Comme le fait justement remarquer Adalsteinsson (1980), le choix entre les différentes hypothèses est pratiquement impossible. C'est pourquoi il a paru intéressant de construire un modèle prenant en compte directement les ségrégations effectives parmi les nouveaux-nés, indépendamment des causes responsables des écarts éventuels entre ces ségrégations et les ségrégations mendéliennes classiques. Un tel modèle doit évidemment tenir compte des différences de fécondité entre les diverses catégories de couples. Après avoir exposé ce modèle, nous tenterons de l'appliquer au cas des chats de l'Ile de Man, en utilisant les données publiées par Todd $(1961,1964)$ et Adalsteinsson (1980).

\section{A. - Modèle général}

\section{1. - Hypothèses}

Chez une espèce à reproduction exclusivement sexuée biparentale, à générations non chevauchantes, une population présente un polymorphisme tel que deux génotypes et deux seulement, $G$ et $G^{\prime}$, sont viables. Soit $\mu$ la fréquence du génotype $G$ parmi les nouveaux-nés d'une certaine génération. Le déterminisme génétique de ce polymorphisme est supposé indépendant du sexe. Pour un nouveau-né, la probabilité d'atteindre l'état d'adulte reproducteur est indépendante du sexe et du génotype (pas de sélection zygotique post-natale, pas de sélection sexuelle). La formation des couples est panmictique. On définit la fécondité d'une catégorie de couples comme l'espérance mathématique du nombre de nouveaux-nés procréés par un couple appartenant à cette catégorie. Les ségrégations peuvent être caractérisées par les valeurs, pour chaque catégorie de couples, de la probabilité $\mathscr{P}(G)$ d'être de génotype $G$ pour un nouveau-né issu d'un couple de la catégorie correspondante. Les notations utilisées sont indiquées dans le tableau ci-dessous :

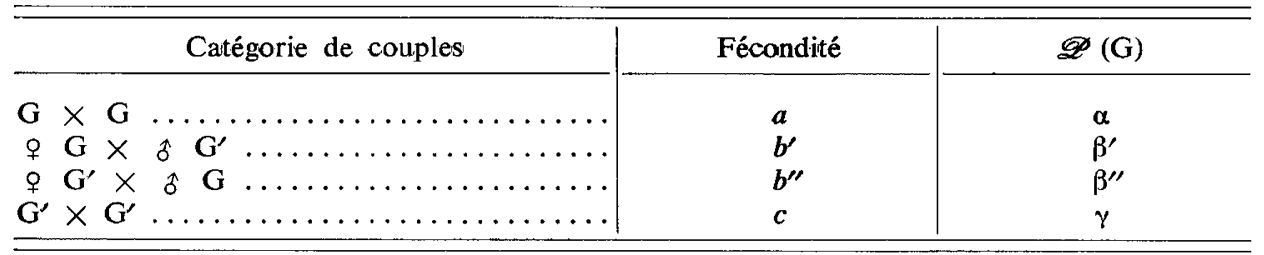

Les paramètres ainsi définis sont supposés constants au cours du temps. Enfin, le modèle élaboré est déterministe, ce qui revient à dire que l'effectif de la population est supposé infiniment grand.

\section{2. - Etablissement d'une relation de récurrence}

En l'absence de sélection post-natale et sous l'hypothèse de la panmixie, les probabilités pour un couple de reproducteurs d'appartenir à chacune des quatre 
catégories sont respectivement $\mu^{2}, \mu(1-\mu), \mu(1-\mu)$ et $(1-\mu)^{2}$. On en déduit la probabilité d'être $\mathrm{G}$ pour un nouveau-né de la génération suivante :

$$
\frac{a \alpha \iota^{2}+\left(b^{\prime} \beta^{\prime}+b^{\prime \prime} \beta^{\prime \prime}\right) \mu(1-\mu)+c \gamma(1-\mu)^{2}}{a \iota^{2}+\left(b^{\prime}+b^{\prime \prime}\right) \mu(1-\mu)+c(1-\mu)^{2}}
$$

L'écriture de cette expression est quelque peu simplifiée si l'on pose $b^{\prime}+b^{\prime \prime}=2 b$ et $\left(b^{\prime} \beta^{\prime}+b^{\prime \prime} \beta^{\prime \prime}\right) /\left(b^{\prime}+b^{\prime \prime}\right)=\beta$ :

$$
\frac{a \alpha \mu^{2}+2 b \beta \mu(1-\mu)+c \gamma(1-\mu)^{2}}{\alpha \mu^{2}+2 b \mu(1-\mu)+c(1-\mu)^{2}}
$$

L'effectif étant infiniment grand, c'est aussi l'expression de la fréquence $\mu^{\prime}$ de $G$ à la génération nouvelle. D'où l'expression de l'accroissement de la fréquence de $G$ d'une génération à la suivante :

$\Delta \mu=\mu^{\prime}-\mu$

$$
=\frac{(2 b-a-c) \mu^{3}+(a \alpha-2 b-2 b \beta+2 c+c \gamma) \mu^{2}+(2 b \beta-c-2 c \gamma) \mu+c \gamma}{a \mu^{2}+2 b \mu(1-\mu)+c(1-\mu)^{2}}
$$

3. - Cas particulier dans lequel le polymorphisme est dî à un couple d'allèles dont l'un est létal à l'état homozygote

Si le polymorphisme est dû à un couple d'allèles autosomal $M-m, M$ étant létal à l'état homozygote, les génotypes $\mathrm{G}$ et $\mathrm{G}^{\prime}$ s'identifient respectivement à $M / \mathrm{m}$ et $m / m$. En supposant l'absence de mutations de $m$ vers $M$, les croisements $m / m \times m / m$ (c'est-à-dire $\left.G^{\prime} \times G^{\prime}\right)$ n'engendrent aucun individu de génotype $M / m$ (c'est-à-dire $G$ ) : donc $\gamma=0$. L'expression de $\Delta \mu$ prend dans ce cas une forme un peu plus simple :

$$
\Delta \mu=\frac{(2 b-a-c) \mu^{2}+(a \alpha-2 b-2 b \beta+2 c) \mu+2 b \beta-c}{a \mu^{2}+2 b \mu(1-\mu)+c(1-\mu)^{2}}
$$

Les structures génotypiques d'équilibre sont données par les solutions de l'équation $\Delta \ell=0$. La solution $\ell=0$ est évidente. Il existe deux autres solutions, les zéros du trinôme du second degré en $\mu$ :

$$
f(\mu)=(2 b-a-c) \mu^{2}+(a \alpha-2 b-2 b \beta+2 c) \mu+2 b \beta-c
$$

Ces deux dernières solutions n'ont de signification biologique que si elles sont réelles et appartiennent à l'intervalle $[0,1]$. Pour la discussion complète, on remarquera que, sur l'intervalle ]0,1], $\Delta \mu$ a toujours le signe de $f(\mu)$. En outre, pour $\mu=1$, $\Delta \mu$ prend la valeur $1-\alpha$, toujours négative (excepté dans le cas particulier, dénué d'intérêt, où $\alpha=1$ ). En résumé, trois situations sont possibles. 


\section{Situation 1}

L'équation $\Delta \mu=0$ admet une solution et une seule appartenant à l'intervalle $] 0,1]$. Cette situation est réalisée si, et seulement si, $2 b \beta-c>0$. Soit $\hat{\mu}$ la solution. $\Delta \mu$ est positif pour $0<\mu<\hat{\mu}$, négatif pour $\hat{\mu}<\mu \leqslant 1$. Donc $\hat{\mu}$ définit un état d'équilibre stable (à condition toutefois qu'en ce point $d(\Delta \mu) / d \mu>-2$ ), atteint asymptotiquement par toute population telle que $\mu$ ne soit pas nulle à la génération origine.

\section{Situation 2}

L'équation $\Delta / \mathfrak{l}=0$ n'admet aucune solution appartenant à l'intervalle $] 0,1]$. Cette situation est réalisée en particulier si $2 b \beta-c<0,2 b-a-c>0$ (les zéros de $f(\mu)$ sont, l'un négatif, l'autre supérieur à 1$)$. Dans tous les cas conduisant à cette situation, $\Delta \mu$ est strictement négatif sur j0,1]. L'état final, atteint asymptotiquement, est l'élimination du génotype $M / m$, donc de l'allèle létal $M$.

\section{Situation 3}

L'équation $\Delta \mu=0$ admet deux solutions appartenant à l'intervalle ]0, 1]. Cette situation n'est réalisable que si $2 b \beta-c<0,2 b-a-c<0$ et pour des valeurs suffisamment grandes de $\alpha$. Si $\hat{\mu}_{1}$ et $\hat{\mu}_{2}$ sont les deux solutions $\left(\hat{\mu}_{1}<\hat{\mu}_{2}\right), \Delta \mu$ est positif pour $\left.\hat{\mu}_{1}<\mu<\right) \hat{\mu}_{2}$, négatif pour $0<\mu<\hat{\mu}_{1}$ et pour' $\hat{\mu}_{2}<\mu<1$. L'état d'équilibre défini par $\hat{\mu}_{1}$ est donc instable; celui que définit $\hat{\mu}_{2}$ est stable si en ce point $\frac{d(\Delta \mu)}{d \mu}>-2$. Le devenir d'une population dépend des conditions initiales : selon qu'à la génération origine $\mu$ est inférieure ou supérieure à $\hat{\mu}_{1}$, on doit assister soit à l'élimination du génotype $M / m$, soit à la stabilisation de sa fréquence à la valeur $\hat{\mu}_{2}$; de toute manière, l'état final est atteint asymptotiquement.

Les trois situations sont illustrées par les graphes de la figure 1.

\section{B. - Application aux chats sans queue de l'Ile de Man}

\section{1. - Estimation des paramètres}

Adalsteinsson (1980) a recensé les résultats de croisements dans lesquels l'un des parents au moins était de génotype $M / \mathrm{m}$. Les croisements $M / \mathrm{m} \times \mathrm{M} / \mathrm{m}$ ont fourni au total 162 individus $M / m$ et 58 individus $m / m$. On en déduit une estimation de $\alpha: 162 / 220 \simeq 0,736$. Les croisements $q \mathrm{M} / \mathrm{m} \times \delta \mathrm{m} / \mathrm{m}$ fournissent 79 individus $M / m$ et 59 individus $m m$, d'où une estimation de $\beta^{\prime}: 79 / 138 \simeq 0,572$. 
Enfin les croisements $q \mathrm{~m} / \mathrm{m} \times \hat{\circ} \mathrm{M} / \mathrm{m}$ fournissent 53 individus $M / m$ et 37 individus $m / m$, d'où une estimation de $\beta^{\prime \prime}: 53 / 90 \simeq 0,589$.

Les fécondités des différentes catégories de couples ont été estimées à partir de données sur le nombre de jeunes par portée publiées par TodD $(1961,1964)$. On a obtenu au total 127 jeunes dans 45 portées issues de croisements $M / m \times M / m$, 86 jeunes dans 27 portées issues de croisements $q M / m, 27$ jeunes dans 6 portées issues de croisements $\circ \mathrm{m} / \mathrm{m} \times \delta \mathrm{M} / \mathrm{m}$ et 128 jeunes dans 33 portées issues de croisements $m / m \times m / m$. Si l'on estime les fécondités par les nombres moyens de jeunes par portée on trouve, respectivement pour $a^{\prime}, b^{\prime}, b^{\prime \prime}$ et $c: 127 / 45 \simeq 2,822$, $86 / 27 \simeq 3,185,27 / 6 \simeq 4,500$ et $128 / 33 \simeq 3,879$.

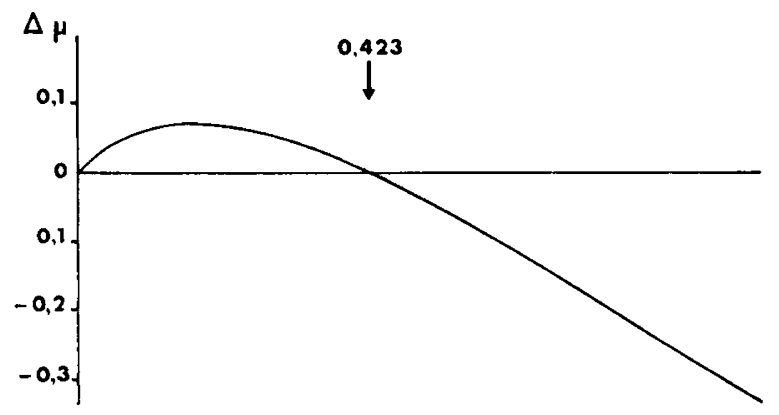

A

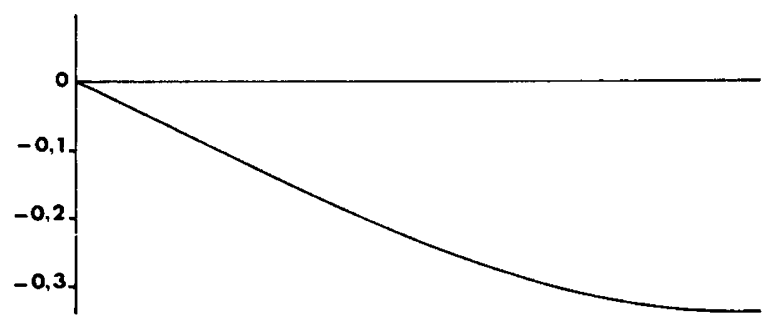

B

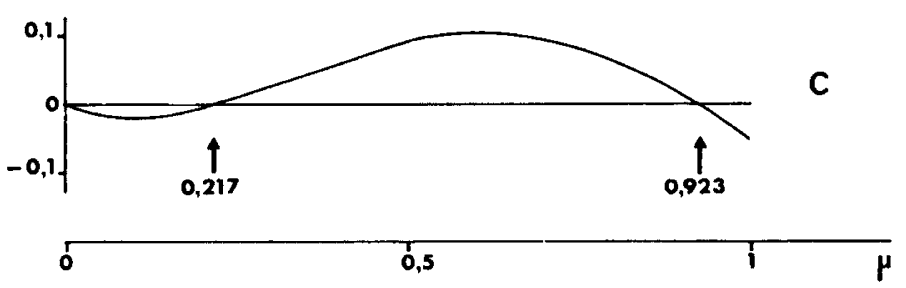

FIG. 1

Graphes représentant les vatiations de $\Delta \mu$ en fonction de $\mu$ dans trois cas particuliers correspondant aux trois situations possibles

Curves presenting the variations of $\Delta \mu$ fonction of $\mu$ in three particular cases corresponding with three possible situations
A : situation $1: a / b=3 / 4 ; a / c=3 / 2 ; \alpha=2 / 3 ; \beta=1 / 2$
B : situation $2: a / b=3 / 4 ; a / c=3 / 4 ; \alpha=2 / 3 ; \beta=1 / 4$
C : situation $3: a / b=3 ; a / c=2 ; \alpha=19 / 20 ; \beta=1 / 2$ 


\section{2. - Etude numérique du modèle}

$\mathrm{Si}$ on reporte ces valeurs dans la formule de $\Delta \mu$ établie plus haut, on obtient :

$$
\Delta \mu=\frac{0,9842 \mu^{2}-2,3228 \mu+0,5946}{-0,9842 \mu^{2}-0,0724 \mu+3,8788} \mu
$$

On voit que c'est la situation 1 qui se trouve réalisée, avec $\widehat{\mu}=0,292$. La figure 2 indique les variations de $\mu$ en fonction du numéro de génération, en supposant que, à la génération origine, $\mu=2.10^{-4}$ (état réalisé si une mutation de $m$ vers $M$ se produit dans une population de 5000 chats, initialement monomorphe). La valeur $\mu=0,1$ est atteinte au bout de 47 générations, 0,2 au bout de 57 générations, 0,290 au bout de 85 générations.

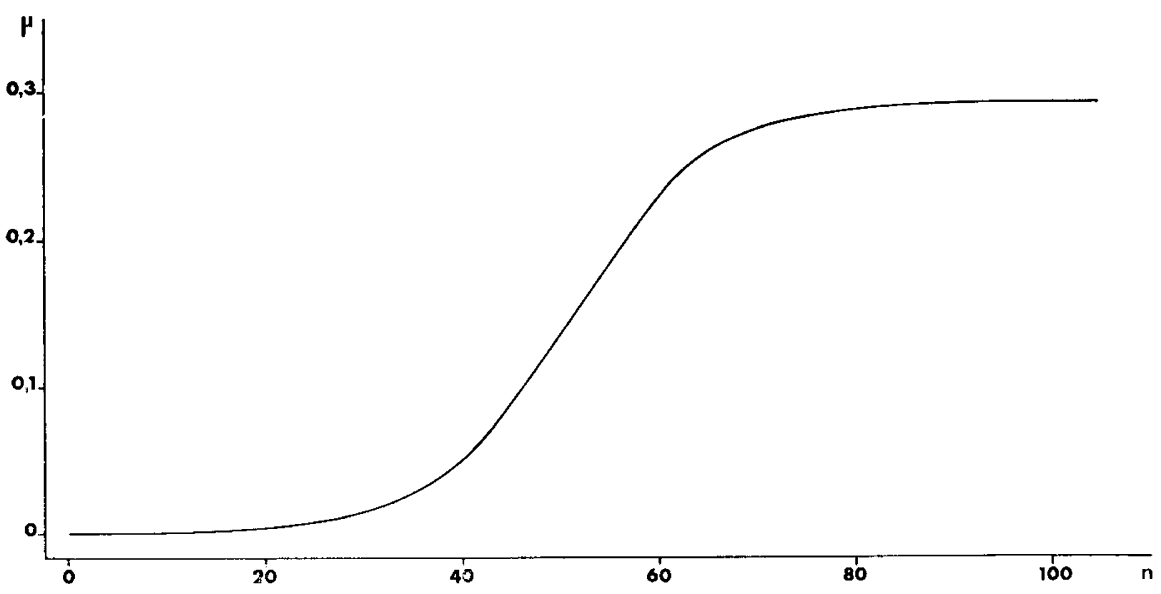

FIG. 2

Variations au cours des générations successives de la fréquence $\mu$ du génotype $\mathrm{M} / \mathrm{m}$ si on donne aux paramètres du modèle les valeurs estimées :

$a=2,82 ; b^{\prime}=3,18 ; b^{\prime \prime}=4,50 ; c=3,88 ; \alpha=0,736 ; \beta^{\prime}=0,572 ; \beta^{\prime \prime}=0,589$

Valeur de $\mu$ à la génération origine : $2.10^{-4}$

Variations along generations of frequency $\mu$ of genotype $\mathrm{M} / \mathrm{m}$ with the following estimated values for the parameters of the model :

$a=2.82 ; b^{\prime}=3.18 ; b^{\prime \prime}=4.50 ; c=3.88 ; \alpha=0.736 ; \beta^{\prime}=0.572 ; \beta^{\prime \prime}=0.589$

The value of $\mu$ at the origin generation is : $2.10^{-4}$

\section{3. - Adéquation aux observations}

Selon Topd et al. (1979), les chats sans queue étaient déjà connus à l'Ile de Man, probablement à fréquence faible, au début du $\mathrm{XIX}^{\circ}$ siècle. Si on suppose que l'intervalle moyen entre générations successives est de 3 ans, cela signifie que 50 générations 
environ se sont écoulées depuis que le génotype $M / m$ a une fréquence non négligeable dans la population. Selon le modèle développé ci-dessus, ce laps de temps permettrait le passage de la fréquence $\mu$ de 0,01 (à la $28^{\mathrm{e}}$ génération) à 0,286 (à la $78^{\mathrm{e}}$ génération) ; cette dernière valeur n'est pratiquement pas différente de la fréquence d'équilibre 0,292. Donc, si le modèle proposé est applicable à la population de l'Ile de Man, celle-ci devrait avoir atteint pratiquement son état d'équilibre.

Or ces mêmes auteurs ont trouvé 69 chats sans queue sur un échantillon de 211 individus, soit une fréquence de 0,327 , très légèrement supérieure à la valeur prédite par le modèle. En fait, la valeur prédite est inévitablement entachée d'erreurs, du fait de l'imprécision des estimations des paramètres du modèle (en particulier, l'estimation de $b^{\prime \prime}$ n'est basée que sur l'observation de 6 portées!). On peut raisonnablement admettre qu'il n'y a pas de désaccord entre les observations et les prédictions du modèle.

\section{4. - Autres modèles possibles}

Adalsteinsson (1980) a construit un modèle fondé sur l'idée que les déviations par rapport aux ségrégations mendéliennes étaient dues à des phénomènes de sélection gamétique et de dérive méiotique. Il estime les paramètres de ce modèle à partir des données de ségrégation utilisées dans le présent travail pour estimer $\alpha, \beta^{\prime}$ et $\beta^{\prime \prime}$. Il prédit l'établissement d'un équilibre dans lequel la fréquence du génotype $M / \mathrm{m}$ est 0,166 . On peut s'étonner de ce qu'il obtienne un résultat aussi différent du nôtre en partant des mêmes données de base. En fait, son modèle pèche sur deux points :

- la population de reproducteurs est supposée avoir à chaque génération une structure génotypique conforme à la loi de Hardy-Weinberg, en excluant le génotype létal $M / M$; on n'a pas le droit de le faire, sélection gamétique et dérive méiotique causant évidemment une déviation de la structure génotypique de la population de zygotes vis-à-vis de la loi de Hardy-Weinberg; cette erreur n'a probablement pas de conséquences considérables;

- les fécondités de tous les croisements sont implicitement considérées comme égales, ce qui est inadmissible : il faudrait au moins prendre en compte le fait que les croisements $\mathrm{M} / \mathrm{m} \times \mathrm{M} / \mathrm{m}$ fournissent moins de descendants que les autres.

On comprend donc que le modèle proposé par Adalsteinsson fournisse des prédictions différentes des nôtres.

En revanche, un autre modèle, simple, peut être proposé. Si on compare les ségrégations observées parmi les produits des croisements $\$ M / m \times \hat{m} / \mathrm{m}$ et o $\mathrm{m} / \mathrm{m} \times$ ô $\mathrm{M} / \mathrm{m}$, on constate qu'elles sont très semblables : $79 \mathrm{M} / \mathrm{m}$ et $59 \mathrm{~m} / \mathrm{m}$ dans le premier cas, 53 et 37 , respectivement, dans l'autre : la différence est loin d'être significative ( $\chi^{2}=0,06$ pour 1 degré de liberté). On s'attendrait à une différence plus importante si ces ségrégations dépendaient de deux mécanismes distincts : dérive méiotique dans le $1^{\text {er }}$ cas, sélection gamétique des spermatozoïdes dans l'autre. Cette remarque conduit à suggérer que l'écart aux proportions mendéliennes proviendrait de la mise en jeu d'un seul et même mécanisme dans les deux types de croisement, le plus simple qu'on puisse envisager étant une survie différentielle des embryons $M / m$ et des embryons $\mathrm{m} / \mathrm{m}$ dans l'utérus maternel. On peut penser que cette survie différentielle existe aussi parmi les produits des croisements $M / m \times M / m$. 
Elle peut être caractérisée par le rapport de la probabilité de survie d'un embryon $m / m$ à celle d'un embryon $M / m$, rapport supposé indépendant du type de croisement ; soit $\sigma$ ce rapport. Si on admet que la population est panmictique, d'effectif infiniment grand et qu'il n'y a aucune autre cause de variation des fréquences alléliques au cours du temps, l'évolution de la population peut être décrite à l'aide d'un modèle classique en génétique des populations, les valeurs sélectives affectées aux génotypes $M / M, M / m$ et $m / m$ étant respectivement 0,1 et $\sigma . S i \mathrm{p}_{\mathrm{n}}$ est la fréquence de l'allèle $M$ à la génération $n$, on $\mathbf{a}$ :

$$
\frac{1}{p_{n}}=\frac{2-\sigma}{1-\sigma}+\sigma^{n}\left(\frac{1}{p_{o}}-\frac{2-\sigma}{1-\sigma}\right)
$$

(voir par exemple GÉNERMONT, 1970)

$p_{n}$ tend asymptotiquement vers $\frac{1-\sigma}{2-\sigma}$, valeur comprise entre 0 et $1 / 2$ si $\sigma<1$,

définissant ainsi un état d'équilibre stable.

On peut aisément estimer $\sigma$ à partir des données de ségrégation, sachant que la probabilité d'être $M / m$ est $\frac{1}{1+\sigma}$ pour un nouveau-né issu de croisement $M / m \times m / m$, 2 $2+\sigma$

méthode du maximum de vraisemblance permet de donner pour $\sigma$ une estimation de 0,72 . L'ensemble des ségrégations observées est en parfait accord statistique avec cette valeur traduisant le même avantage sélectif des zygotes $M / m$ quel que soit le type de croisement $\left(\chi^{2}=0,07\right.$ pour 2 degrés de liberté).

$\mathrm{Si}$ on retient cette valeur de $\sigma$, la valeur limite de $\mathrm{p}_{\mathrm{n}}$ prédite par le modèle est 0,217 , soit pour $\mu$ une valeur limite de 0,43 , nettement supérieure à la valeur observée dans la population actuelle, alors que, selon le modèle l'état d'équilibre est pratiquement atteint en moins de 50 générations si à la génération origine $u$ a la valeur $2.10^{-4}$. Ce modèle classique ne semble donc pas décrire de façon satisfaisante la situation réelle.

\section{Conclusions}

Des trois modèles envisagés, celui dont les prédictions s'accordent le mieux aux observations est celui qui a été développé dans la première partie de cet article. Sa supériorité provient pour une large part du fait qu'il utilise deux catégories de paramètres, ceux qui décrivent les ségrégations, et ceux qui décrivent les fécondités. Au contraire, le modèle d'Adalsteinsson suppose égales les fécondités de tous les types de couples et le modèle classique qui vient d'être présenté utilise un seul paramètre $(\sigma)$ pour caractériser à la fois les ségrégations et les différences de fécondité. 
Le modèle que nous préconisons est un modèle robuste, car il ne repose sur aucune hypothèse relative aux causes des ségrégations ou des différences de fécondité. Ses paramètres sont donc objectifs; de plus, ils sont aisément estimés à partir des résultats de croisements. Il peut être adapté sans grande difficulté à toutes les situations dans lesquelles il n'y a pas de sélection post-natale.

En ce qui concerne plus particulièrement le cas du maintien du gène $M$ chez les chats de l'Ile de Man, il est clair qu'on peut décrire les phénomènes actuellement connus sans prendre en compte aucune sélection post-natale. Il ne faudrait toutefois pas considérer comme démontrée l'absence d'une telle sélection. On peut très bien envisager l'existence de plusieurs formes de sélection post-natale dont les effets se compensent approximativement. Il est en effet bien connu que l'absence (ou plutôt la très forte réduction) de la queue est accompagnée d'anomalies graves (voir par exemple JAMES et al., 1969) : il ne serait pas étonnant que ces anomalies entraînent une faible compétitivité des animaux $M / m$ vis-à-vis des $m / m$. Ce défaut de compétitivité naturelle pourrait être compensé par une intervention humaine visant à conserver préférentiellement un phénotype exceptionnel. Un tel mécanisme ne pourrait être mis en évidence que par une étude précise des aptitudes des génotypes $M / m$ et $\mathrm{m} / \mathrm{m}$ (comparaison des espérances de vie par exemple) ainsi que du comportement des propriétaires de chats de l'Ile de Man.

Reçu pour publication en octobre 1981.

\author{
Summary \\ A lethal gene maintained in a population: \\ the case of the tailless cat of the Isle of Man
}

A deterministic model is presented, accounting for the maintenance of a lethal allele in a population on the basis of distorsions of mendelian ratios associated with differential fecundity. The predictions of the model are discussed with special reference to the population of domestic cats of Isle of Man : the maintenance of the $M$ allele (determining taillessness in heterozygous state) may be understood without postulating any kind of postnatal zygotic selection.

\title{
Références bibliographiques
}

AdALSTEINSSON S., 1980. Establishment of equilibrium for the dominant lethal gene for Manx taillessness in Cats. Theor. appl. Genet., 58, 49-53.

Genermont J., 1970. Recueil d'exercices de génétique des populations. Masson, Paris.

JAMES C.C.M., Lassman L.P., Tomlinson B.E., 1969. Congenital anomalies of the lower spine and spinal cord in Manx cats. J. Pathol., 97, 269-276.

Todn N.B., 1961. The inheritance of taillessness in Manx cats. J. Hered., 52, 228-232.

Tond N.B., 1964. The Manx factor in domestic cats. J. Hered., 55, 225-230.

Todd N.B., Garrad L.S., Blumenberg B., 1979. Mutant allele frequencies in domestic cats of the Isle of Man. Carniv. Genet. Newsl., 3, 388-407. 